\title{
Duration compression induced by visual and phonological repetition of Chinese characters
}

\author{
Lina $\mathrm{Jia}^{1} \cdot$ Zhuanghua $\mathrm{Shi}^{2}$
}

Published online: 27 June 2017

(C) The Psychonomic Society, Inc. 2017

\begin{abstract}
Our prior experience heavily influences our subjective time. One of such phenomena is repetition compression, that is, repeated stimuli are perceived shorter than novel stimuli. However, most of the studies on repetition compression used identical stimuli, leaving the question whether similar repetition effects could take place in phonological and semantic level repetition. We used Chinese characters to manipulate different levels of repetition in a duration discrimination task. We replicated earlier findings that repetition of visual identical characters shortened the apparent duration and found the repetition compression was spatially independent. Phonological repetition also caused the duration compression though the effect was weaker than the visual repetition. However, we observed no duration compression during the semantic repetition. The results suggest that repetition compression is mediated by visual and phonological representation of a stimulus in an early stage in processing hierarchy. We explained our findings according to the framework of predictive coding.
\end{abstract}

Keywords Duration judgment $\cdot$ Repetition compression · Subjective time

Electronic supplementary material The online version of this article (doi:10.3758/s13414-017-1360-3) contains supplementary material, which is available to authorized users.

Zhuanghua Shi

strongway@psy.lmu.de

1 Department of Education, School of Humanities, Jiangnan University, Wuxi, China

2 General and Experimental Psychology, Department of Psychology, Ludwig-Maximilians-Universität München, Munich 80802, Germany

\section{Introduction}

We all experience time flies and time drags. Our subjective time is susceptible to many factors, such as nontemporal properties, attention, emotion, action and intention, and prior experience (Allman, Teki, Griffiths, \& Meck, 2014; Droit-Volet \& Gil, 2009; Ganzenmüller, Shi, \& Müller, 2012; Jia, Shi, \& Feng, 2015; Matthews \& Meck, 2016; Shi \& Burr, 2016; Shi, Church, \& Meck, 2013; Shi, Ganzenmüller, \& Müller, 2013; Xuan, Zhang, He, \& Chen, 2007). The latter, prior experience, causes various types of time distortions, such as spatialspecific duration compression (Johnston, Arnold, \& Nishida, 2006), central tendency of duration judgment (Gu, Jurkowski, Shi, \& Meck, 2016; Jazayeri \& Shadlen, 2010; Shi et al., 2013), odd-ball time dilation (Pariyadath \& Eagleman, 2007; Tse, Intriligator, Rivest, \& Cavanagh, 2004), and repetition compression (Birngruber, Schröter, \& Ulrich, 2015b; Matthews, 2011; Matthews \& Gheorghiu, 2016). One remarkable example is that repeating of the first item only once suffices to shortens the apparent duration (Birngruber et al., 2015b; Matthews, 2011), while the first item in a train of repeated displays seems longer than the subsequent items (Rose \& Summers, 1995).

Studies have addressed the effect of repetition compression on subjective time by manipulating multiple factors, such as repetition times, repetition probability, novelty, and spatial location (Birngruber, Schroter, \& Ulrich, 2015a; Cai, Eagleman, \& Ma, 2015; Pariyadath \& Eagleman, 2007; Pariyadath \& Eagleman, 2008, 2012; Schindel, Rowlands, \& Arnold, 2011). Using a stream-based oddball paradigm, Pariyadath and Eagleman (2007, 2008, 2012; Pariyadath \& Eagleman, 2007; Pariyadath \& Eagleman, 2008, 2012) showed that the duration compression of the repeated standard stimuli positively correlates with the number of repetition in the stream and the novelty of the oddball (but see Birngruber 
et al., 2015a). Some suggest this duration compression is related to repetition suppression, which assumes repeated signals reduce neural responses (Grill-Spector, Henson, \& Martin, 2006; Henson \& Rugg, 2003). This also is in line with the predictive coding, which suggests that the brain do little when the incoming stimulus matches the internal expectation but passes prediction errors for further processing when the incoming signal deviates from the prediction (Eagleman \& Pariyadath, 2009; Friston, 2005; Rao \& Ballard, 1999; Shi \& Burr, 2016). However, a recent study by Matthews (2015) reported that the repetition compression of subjective duration is reduced and even reversed when repetition trials become more frequent. The finding suggests there is an interaction between the repetition suppression and the attentional improvement of relevant informative processing modulated by the same high-level expectation (Matthews \& Meck, 2016). Studies also showed the temporal repetition compression can be spatial specific (Burr, Tozzi, \& Morrone, 2007; Cai et al., 2015), consistent with the notion of the low-level adaptive processing (Johnston et al., 2006). For instance, Cai et al. (2015) found that the repetition compression effect diminished when a repeated comparison stimulus (a bar with the same orientation) was at a different location. These results indicate that the temporal processing is similar for a repeated stimulus compared to an irrelevant (novel) item when the standard and comparison are at different locations.

Earlier research, however, primarily focused on the repetition effect induced by repeating simple visual or auditory items (Birngruber et al., 2015b; Kim \& McAuley, 2013; Matthews \& Gheorghiu, 2016; Matthews \& Meck, 2016). Complex stimuli, such as Chinese characters, not only appear as visual patterns of strokes but also invoke high-level phonological and semantic cognitive processing. An interesting question arises about whether repeating nonvisual constituents of a visual item, such as phonological or semantic repetition of a Chinese character, also could compress apparent duration. Research has shown that phonological or semantic repetition can facilitate cognitive processes of Chinese characters in various tasks, such as identification, categorization, lexical decision, and naming task (Neely, 1991; Perfetti \& Liu, 2006). The facilitation by phonological or semantic repetition is correlated with reduced neural activities (Qiao, Zheng, Li, Zhu, \& Wang, 2014; Schacter, Wig, \& Stevens, 2007; Sekiguchi, Koyama, \& Kakigi, 2000; Takahiro, Sachiko, \& Ryusuke, 2004; Wheatley, Weisberg, Beauchamp, \& Martin, 2005). Similar neural repetition suppression has been proposed to explain the temporal repetition compression (Matthews \& Gheorghiu, 2016; Pariyadath \& Eagleman, 2007).

Chinese characters have their own visual shapes, pronunciations, and semantic meanings, which allows us to manipulate repetition in different levels. For example, two Chinese characters, 目vs., are homophones with no visual similarity or semantic links ("eye" and "wood") but share the same pronunciation $(/ \mathrm{mu} /)$. Another example, Chinese characters, 足 $(/ \mathrm{zu} /)$ and 脚 $(/ \mathrm{jiao} /)$, have the same semantic meaning "foot" but share no visual or phonological similarity. We hypothesized that if repetition effect occurs at any levels of temporal processing, subjective duration would be compressed for visual, phonological, and semantic repeated characters. If the repetition compression limits to the low-level process, we expect the time compression in visual repetition, but not on the high-level repetition. In addition, if the repetition effect is location-specific (Burr et al., 2007; Cai et al., 2015; Johnston et al., 2006), changing the location of the second item would null the compression effect.

To test the above hypotheses, we used two-interval duration discrimination task in which a standard and a comparison durations were shown sequentially, and participants judged whether the comparison was shorter or longer than the standard one. To address if the effect of repetition is locationspecific, the standard and comparison were at the same or separate locations in Experiment 1. In Experiments 2 and 3 we tested whether phonological repetition and semantic repetition would also compress apparent duration.

\section{Experiment 1}

\section{Method}

\section{Participants}

Twenty-four Chinese volunteers (15 females; mean age 22 years; 12 in each sub-experiment) took part in the experiment. All had normal or corrected-to-normal vision and were naïve to the purpose of the experiment. All participants gave informed consent before the experiment.

\section{Stimuli and apparatus}

The experiment took place in a sound-isolated cabin with a dimly luminance. The critical visual stimuli were Chinese characters, including two groups (Groups A and B) with equal frequencies (mean 419 characters/million), and the mean of strokes was 7.5 (Cai \& Brysbaert, 2010). Each group includes 77 characters. For half of the participants, the characters from Group A were standard stimuli and the characters from Group B were comparison stimuli and vice versa for the other half of participants. The white characters (about $1.8^{\circ}$ of viewing angle) were presented on a black background using a 24-inch BenQ Gaming LCD monitor (1 ms fast response time) with a resolution of $1,024 \times 867$ pixels and the refresh rate of $100 \mathrm{~Hz}$. The viewing distance was $57 \mathrm{~cm}$. Participants judged the duration of characters by pressing left- and right- arrow key on a standard keyboard. The experiment was controlled by self- 
coded Matlab program with the support of Psychophysics Toolbox (Brainard, 1997).

\section{Design and procedure}

The experiment used the classic duration comparison paradigm. Each trial comprised a standard and comparison durations. The standard and comparison durations were the presentation time of Chinese characters on the screen. Both the standard and comparison, either the same or different characters, were presented sequentially in the center of the screen in Experiment 1a. In Experiment 1b, the standard and comparison were randomly presented at two possible horizontal locations - one left and one right — at $5^{\circ}$ eccentricity (Fig. 1). Thus, there were four experimental conditions: 1) the standard and comparison were on a same location with a repeated character ("sLrC"); 2) the standard and comparison were on a same location but with nonrepeated characters ("sLnC"); 3) the standard and comparison were on different locations with a same character ("dLrC"); 4) the standard and comparison were on different locations with non-repeated characters
("dLnC"). To reduce influence of eye movements on temporal judgment, we asked participants to avoid any saccades during the stimulus presentation.

Figure 1 illustrates two typical trial presentations for Experiment $1 \mathrm{a}$ and $1 \mathrm{~b}$, respectively. A trial started with a fixation cross in the center of the screen. In Experiment 1a, the fixation cross lasted for $800 \mathrm{~ms}$ followed by a blank screen of 500 to $800 \mathrm{~ms}$. In Experiment 1b, the fixation cross lasted 500 to $800 \mathrm{~ms}$ before the first standard stimulus and remained on the screen until the response. The first standard with a fixed duration of $600 \mathrm{~ms}$ was presented, which followed by a random interstimulus interval (ISI) of 300 to $500 \mathrm{~ms}$. The second comparison was shown for a duration randomly selected from 300, 400, 500, 600, 700, 800, and $900 \mathrm{~ms}$. After a 500-ms blank screen, a question mark prompted for a response. Participants had to judge whether the comparison was shorter or longer than the standard by pressing either the left- or the right-arrow key.

All experimental conditions were within-subject design in a random order. There were 7 experimental blocks of 44 trials in Experiment 1a and 14 experimental blocks of 44 trials in

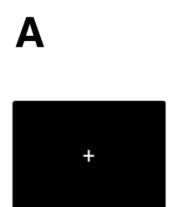

$800 \mathrm{~ms}$

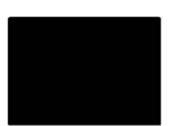

$500-800 \mathrm{~ms}$

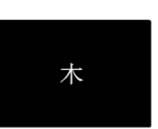

$600 \mathrm{~ms}$

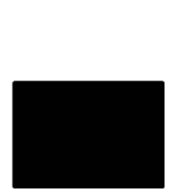

$300-500 \mathrm{~ms}$

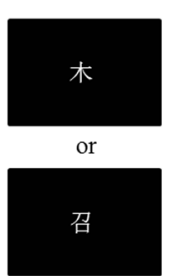

$300,400,500,600$ 700,800 , and $900 \mathrm{~ms}$

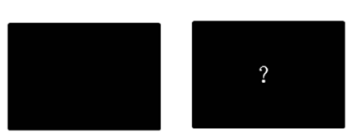

$500 \mathrm{~ms}$

time

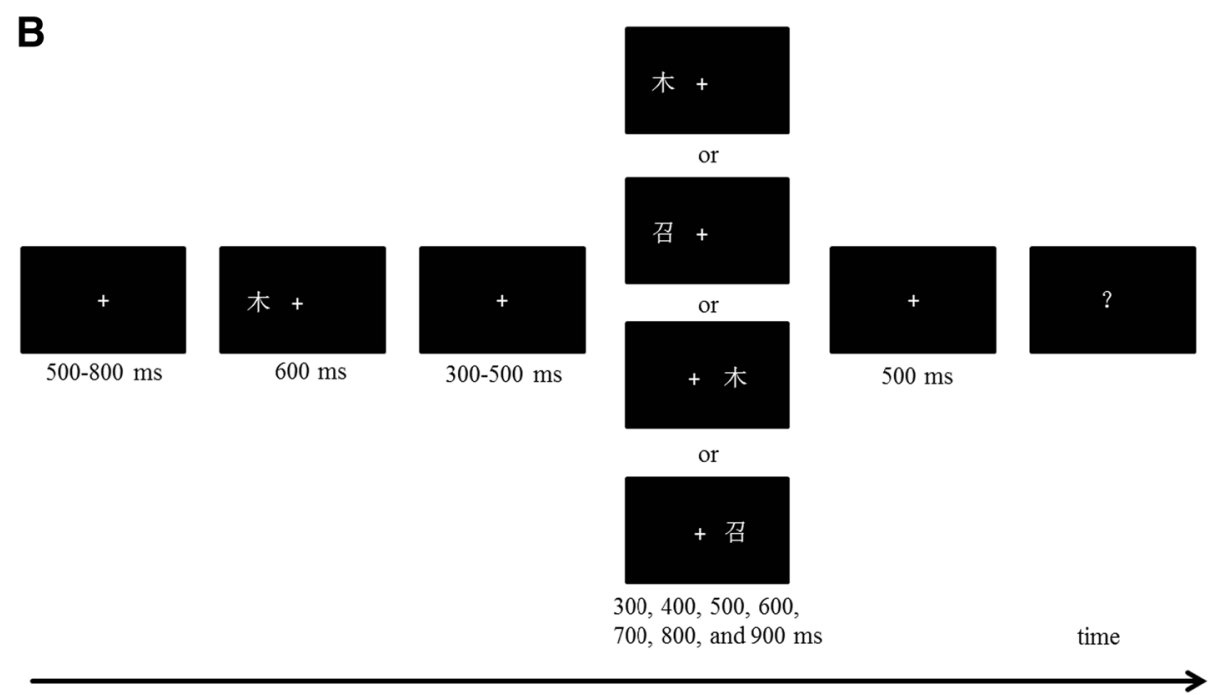

Fig. 1 Schematic illustrations of stimuli presentation in Experiment 1. (a) The first (standard) and second (comparison) characters were both presented in the center of the screen, and the comparison character (e.g., 木 $/ \mathrm{mu} /$, meaning “wood”) could be the same character (木) or a novel one

(召/zhao/, meaning such as “call”) in Experiment 1a. (b) The comparison character was displayed either in the same location or opposite locations with the same or a different character as the standard one in Experiment $1 b$ 
Experiment $1 \mathrm{~b}$. The intertrial interval was $2,000 \mathrm{~ms}$. Participants could take a break between blocks. Each experimental condition repeated 22 times. Before the formal experiment, each experiment included 2 practice blocks of 28 trials.

\section{Data analysis}

Repeated-measures ANOVAs and Bayes Factors (BF) analyses were conducted for each experiment. Bayes Factors with default prior scales were calculated for those marginal and nonsignificant results to show any evidence for supporting the alternative or the null hypothesis (Rouder, Morey, Speckman, \& Province, 2012).

To estimate psychometric functions, the proportions of "longer" judgments were plotted as a function of the comparison duration and fitted with a logistic regression for each experimental condition and each participant. From the fitted curves, the 50\% threshold, at which participants made equal "longer" and "shorter" judgments, is referred as the point of subjective equality (PSE) (Jia, Shi, Zang, \& Müller, 2013; Shi, Jia, \& Müller, 2012; Treutwein \& Strasburger, 1999). Similarly, the just-noticeable difference (JND), as an index of discrimination sensitivity, was calculated by estimating half the difference in duration between the $25 \%$ and $75 \%$ thresholds.

\section{Results}

We first examined if there were any systematic response biases in the behavioral data, which could be identified by the discrimination errors in the two extreme comparison durations (300 and $900 \mathrm{~ms}$ ). If there were any systematic response biases, the discrimination errors would be systematically varied across conditions. On average, the discrimination error rates were very low, 0.025 and 0.01 for the repeated and novel conditions in Experiment 1a, and 0.013, 0.006, 0.019, and 0.006 for the sLrC, sLnC, dLrC, and dLnC conditions in Experiment $2 \mathrm{~b}$. Bayesian Repeated Measures ANOVAs failed to reveal any significant difference between the repeated and novel conditions $(\mathrm{BF}=0.478)$ in Experiment 1a, and strong evidence that discrimination errors did not differ among the four conditions ( $\mathrm{BF}=0.297)$ in Experiment $2 \mathrm{~b}$. The findings suggest response biases, if any, could be neglected from further analyses.

Figure 2 shows the estimated psychometric curves for the repeated and novel conditions in Experiment 1a. The mean PSEs $( \pm \mathrm{SE})$ were $613 \pm 24$ and $567 \pm 18 \mathrm{~ms}$ for the repeated and novel conditions, respectively. A one-way repeated-measures ANOVA showed that the mean PSE was significantly higher for the repeated than the novel condition, $F(1,11)=$ 6.94, $p<0.05, \eta_{\mathrm{p}}{ }^{2}=0.39$, showing a shortened perceived duration for the repeated character than the novel character. The finding is consistent with the literature (Birngruber et al., 2015b; Matthews, 2011; Matthews \& Gheorghiu, 2016). The discrimination sensitivities, measured by the JNDs, failed to

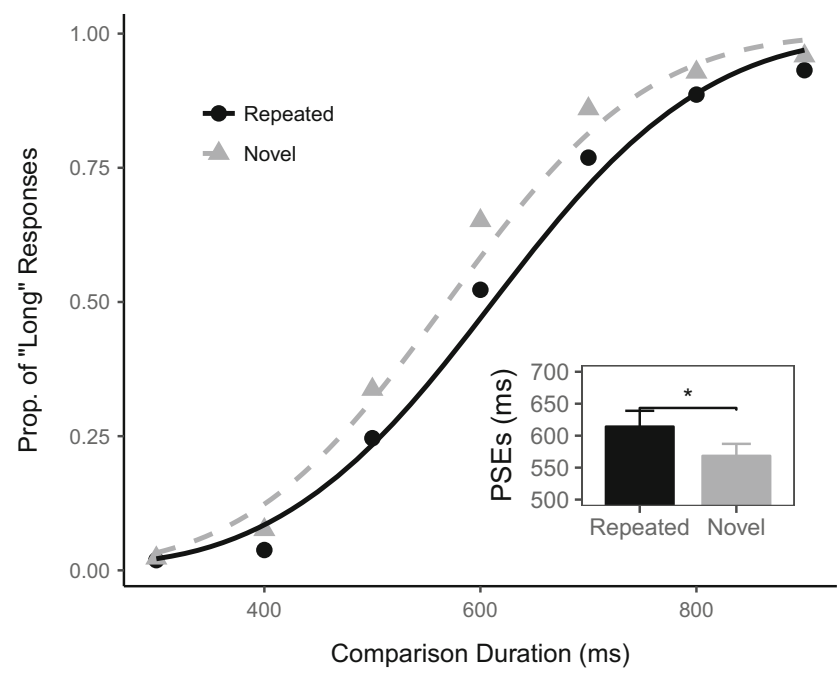

Fig. 2 Results of Experiment 1a. Mean proportions of "long" responses are plotted against the comparison durations with the fitted psychometric curves, respectively, for the repeated and novel conditions. The inset depicts the mean PSEs and the related standard errors for the two conditions $(* p<0.05)$

find any difference between two conditions, $F(1,11)=1.41, p$ $=0.26, \eta_{\mathrm{p}}{ }^{2}=0.11, \mathrm{BF}=0.59$.

Figure 3 shows the results of Experiment $1 \mathrm{~b}$. The mean PSEs $( \pm$ SE) were $592 \pm 14,562 \pm 11,576 \pm 18$, and $552 \pm$ $16 \mathrm{~ms}$ for the $\mathrm{sLrC}, \mathrm{sLnC}, \mathrm{dLrC}$, and $\mathrm{dLnC}$ conditions, respectively. Two-way repeated-measures ANOVA of PSEs with location (same vs. different) and repetition (repeated vs. novel) as factors showed a significant higher PSE for the repeated $(584 \mathrm{~ms})$ than the novel $(557 \mathrm{~ms})$ conditions, $F(1,11)$ $=24.32, p<0.001, \eta_{\mathrm{p}}{ }^{2}=0.69$, consistent with the finding of Experiment 1a. However, there were no significance for the main effect of the location, $F(1,11)=1.57, p=0.24, \eta_{\mathrm{p}}{ }^{2}=$ $0.13, \mathrm{BF}=0.763$, and the interaction between two factors, $F$ $(1,11)=0.41, p=0.54, \eta_{\mathrm{p}}{ }^{2}=0.04, \mathrm{BF}=0.386$. The finding showed the repetition compression was stable even when the standard and comparison were in different location. Same as in Experiment 1a, the discrimination sensitivities (i.e., JND) between the repeated and novel conditions were not different, $F(1,11)=0.15, p=0.71, \eta_{\mathrm{p}}{ }^{2}=0.01$, supported by a positive evidence from a Bayes factor of 0.291. However, presenting the standard and comparison stimuli at different location worsened the discrimination sensitivity, as shown by the main effect of location on JND just reaching significance with a positive evidence, $F(1,11)=4.72, p=0.05, \eta_{\mathrm{p}}{ }^{2}=0.30$, BF $=4.23$, which might be caused by attentional shift between two locations. Of note, the attentional shift did not alter the repetition compression effect shown in the PSEs. The interaction between the repetition and location was not significant on JNDs, $F(1,11)=1.79, p=0.21, \eta_{\mathrm{p}}{ }^{2}=0.14, \mathrm{BF}=0.595$.

Taken together, the findings revealed that the subjective duration of the comparison was shortened when it was a repeated character compared to a novel character. 


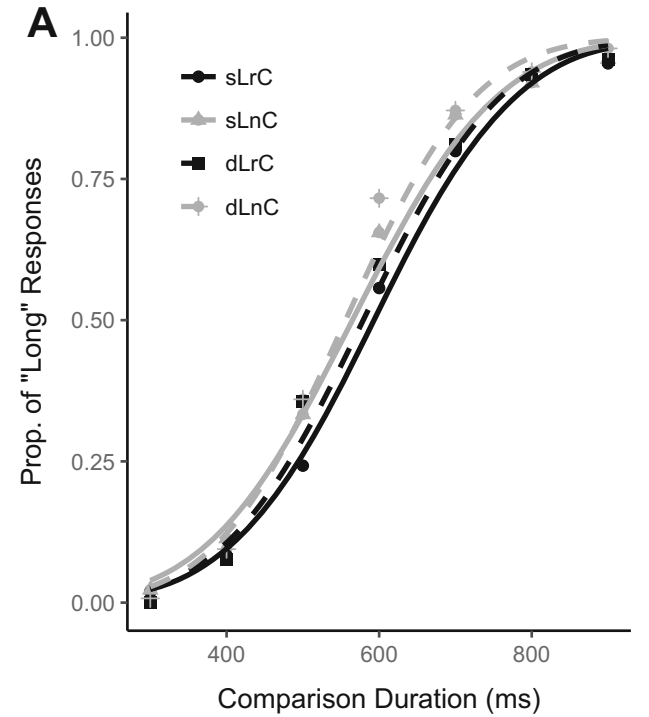

Fig. 3 Results of Experiment 1b. (a) Mean proportions of "long" responses are plotted against the comparison durations with the fitted psychometric curves for the four experimental conditions. (b) The mean PSEs and the related standard errors are shown for the four experimental

Unlike the adaptation effect (Burr et al., 2007; Cai et al., 2015; Johnston et al., 2006), this repetition compression shown in the comparison task was not location specific. To examine whether the repetition compression can take place at high-level cognitive processes, we compared the visual repetition, phonological repetition to the non-repetition baseline in Experiment 2.

\section{Experiment 2}

\section{Method}

\section{Participants}

Ten Chinese volunteers ( 4 females, mean age 21.8 years) took part in this experiment. All had normal or corrected-to-normal vision and were naïve about the purpose of the experiment. They all gave informed consent prior to the experiment.

\section{Stimuli and apparatus}

The stimuli presentation and apparatus were the same to those in Experiment 1, except that this experiment used three groups with equal frequencies (mean 381 characters/million) and their mean number of strokes was 7.7. Each group includes 77 characters. Among them, two groups of characters were homophones but sharing no visual or semantic similarity (see supplementary Table 1).

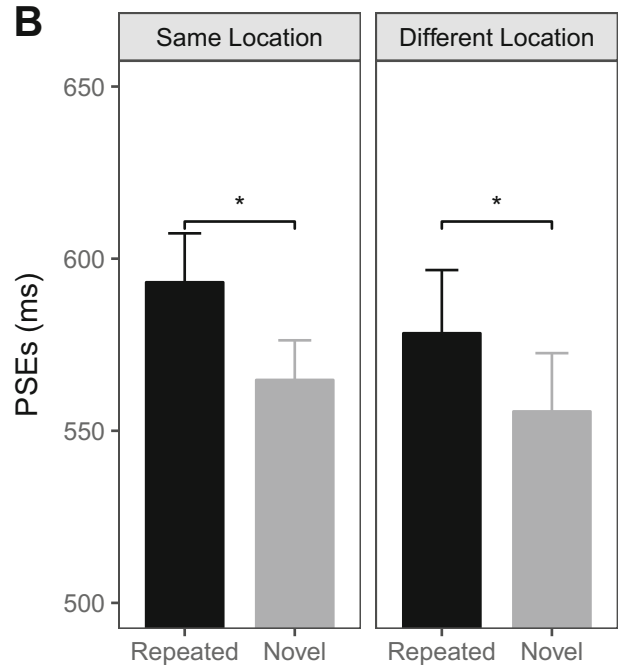

conditions. sL, same location; dL, different location; rC, repeated character for the comparison stimulus; $\mathrm{nC}$, novel character for the comparison stimulus $(* p<0.05)$

\section{Procedure}

The experimental set-up was the same as used in Experiment $1 \mathrm{a}$, with the following exceptions. There were three conditions: the visual repetition (VR), phonological repetition $(\mathrm{PR})$, and novel $(\mathrm{N})$ conditions. The standard and comparison used the same character in the VR condition whereas they shared the same pronunciation (homophones) but different characters in the PR condition (e.g., 目 $/ \mathrm{mu} /$ and 木 $/ \mathrm{mu} /$ ). The novel baseline condition used different characters that differ in both shape and pronunciation (e.g., 目 $/ \mathrm{mu} /$ and朵 /duo/). The experiment comprised 1 practice block and 11 experimental blocks, with each of 42 trials. Each combinational experimental condition repeated 22 times, presented in a random order.

\section{Results}

The discrimination error rates calculated from the two extreme comparison durations were very row, $0.019,0.007$, and 0.013 for the visual-repetition, phonological-repetition, and novel conditions. Bayesian repeated measures ANOVA failed to reveal any significance among the three conditions $(B F=0.826)$. That is, we did not observe any significant response biases.

Figure 4 depicts the results of Experiment 2. The mean PSEs $( \pm$ SE) were $592 \pm 18,556 \pm 24$, and $534 \pm 22 \mathrm{~ms}$ for the visual-repetition, phonological-repetition, and novel conditions, respectively. The ANOVA revealed a significant effect of repetition on the subjective duration with GreenhouseGeisser correction, $F(1.303,11.731)=11.11, p<0.01, \eta_{\mathrm{p}}{ }^{2}=$ 0.55 . Follow-up Holm-Bonferroni correction revealed that the mean PSEs was significantly higher for the visual-repetition 


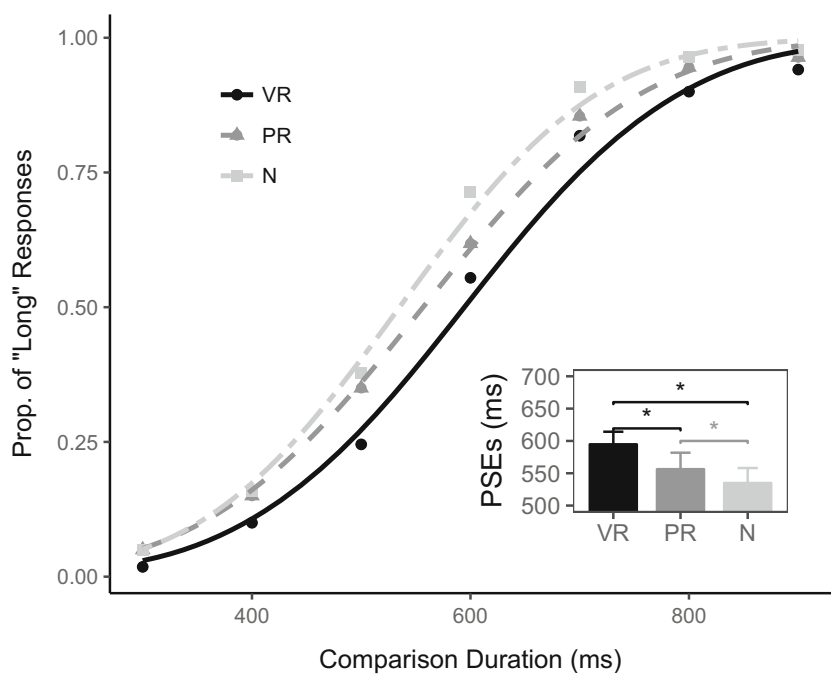

Fig. 4 Results of Experiment 2. Mean proportions of "long" responses are plotted against the comparison durations with the fitted psychometric curves for the conditions of visual-repetition (VR), phonologicalrepetition (PR), and novel $(\mathrm{N})$ characters, respectively. The inset shows the mean PSEs and the related standard errors for the three conditions $\left({ }^{*} p<0.05\right)$

condition compared with the phonological-repetition and novel conditions (mean differences: $36 \mathrm{~ms}$, corrected $p=0.02$; and $58 \mathrm{~ms}$, corrected $p=0.02$ ), and significantly higher for the phonological-repetition than the novel condition (mean difference: $22 \mathrm{~ms}$, corrected $p=0.03$ ). The JNDs, however, failed to find any difference among the three experimental conditions, $F(2,18)=2.58, p=0.10, \eta_{\mathrm{p}}{ }^{2}=0.22, \mathrm{BF}=0.95$. The findings confirmed that the visual repetition shortens the perceived duration. In addition, we showed that the phonological repetition could shrink perceived durations, although the effect was weaker than the pure visual repetition. In Experiment 3, we further examined whether semantic repetition, which involves high-level cognitive process, could still shorten the apparent duration.

\section{Experiment 3}

\section{Method}

\section{Participants}

Ten Chinese volunteers ( 6 females, mean age 21.5 years) took part in this experiment. All had normal or corrected-to-normal vision and were naive to the purpose of the experiment. They all gave informed consent before the experiment.

\section{Stimuli and apparatus}

The stimuli presentation and apparatus were the same as in Experiment 1a, except the followings. In this experiment, 66 characters (mean of strokes 8.7) with equal frequencies (mean 400 characters/million) were used. Twenty-two pairs of characters have close semantic meanings (see Table 1 in the Appendix), whereas the rest of the 22 characters have complete different semantic meanings. Each semantic pair was tested for all comparison durations (i.e., from 300 to $900 \mathrm{~ms}$ ), such that individual variability of semantic processing among semantic pairs, if any, would not influence a particular test duration.

Following the formal test, participants had to rate the semantic similarity of the presented characters using a 5-point scale rating (" 1 " means two characters are semantic irrelevant, and " 5 " semantic highly relevant).

\section{Procedure}

The experimental procedure was the same as in Experiment 2, except the phonological-repetition condition replaced by the semantic-similarity (SS) condition (e.g., 足 /zu/ and 脚 /jiao/ both have the same semantic meaning of foot). The experiment included 2 practice blocks with each of 42 trials and 7 experimental blocks with each of 66 trials.

\section{Results}

Like the previous two experiments, the discrimination error rates calculated from the two extreme comparison durations were row, 0.036, 0.002, and 0.006 for the visual-repetition, semantic-similarity, and novel conditions. Bayesian repeated measures ANOVA failed to reveal any significance among the three conditions $(\mathrm{BF}=0.854)$.

The mean PSEs $( \pm$ SE) were $647 \pm 19,573 \pm 12$, and $566 \pm$ $15 \mathrm{~ms}$ separately for the visual-repetition, semantic-similarity and novel conditions (Fig. 5). A one-way repeated-measures ANOVA revealed that the PSEs among three repetition conditions differed significantly, $F(2,18)=26.30, p<0.001, \eta_{\mathrm{p}}{ }^{2}=$ 0.75 . The post-hoc Holm-Bonferroni correction confirmed that the mean PSEs were significantly higher for the VR condition than for the SS and N conditions (mean differences: $74 \mathrm{~ms}$, corrected $p=0.002$; and $81 \mathrm{~ms}$, corrected $p=0.0001$ ), which was consistent with the findings of Experiments 1 and 2 . Interestingly, we failed to find any significant difference (mean difference: $7 \mathrm{~ms}$, corrected $p=0.29, \mathrm{BF}=0.599$ ) between the semantic-similarity and the novel conditions. Merely semantic repetition failed to distort the perceived duration. This contrasts with the finding of Experiment 2, in which the phonological repetition caused a duration compression. In addition, the main effect of repetition on JND was not significant, $F(2,18)=3.42$, $p=0.06, \eta_{\mathrm{p}}^{2}=0.28, \mathrm{BF}=1.631$.

In addition, the mean rated ( $\pm \mathrm{SE}$ ) for the semantically similar judgment was $4.2( \pm 0.11)$, indicating the characters used in the SS condition were highly relevant in semantic. 


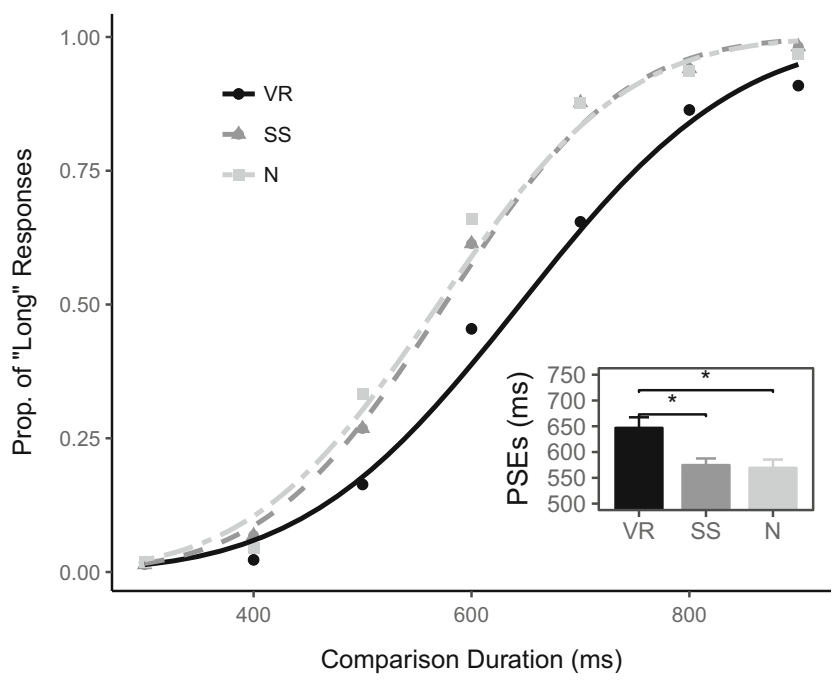

Fig. 5 Results of Experiment 3. Mean proportions of "long" responses are plotted against the comparison durations with the fitted psychometric curves for the visual-repetition (VR), semantic-similarity (SS), and novel $(\mathrm{N})$ conditions, respectively. The inset depicts the mean PSEs and the related standard errors for the two conditions $\left({ }^{*} p<0.05\right)$

\section{General discussion}

The present study investigated influences of different level of repetition on duration estimation using Chinese characters. We compared three levels of repetition: the visual (e.g., 木- ), phonological (e.g., 目/mu/- /mu/), and semantic (e.g., 足/zu/ - 脚/ jiao/) repetitions. Our findings on the visual repetition replicated the repetition compression effect found in the literature (Birngruber et al., 2015b; Cai et al., 2015; Matthews, 2011; Matthews \& Gheorghiu, 2016; Matthews \& Meck, 2016). We further showed that the repetition compression was location independent (Experiment 1b). More interestingly, the highlevel phonological repetition also shortened the apparent duration, but the compression effect was weaker than the pure visual repetition (Experiment 2). However, the repetition compression diminished when the repetition was on the semantic level (Experiment 3). Among all three experiments, we did not observe any systematic response biases, which suggests the compression effect we found here is unlikely a pure response bias.

Our finding of location-independent repetition compression (Experiment $1 b$ ) differs from earlier findings using simple visual stimuli (Cai et al., 2015). Repetition of simple visual stimuli (such as Gabor patch) likely invokes low-level retinotopic specific adaptation (Ayhan, Bruno, Nishida, \& Johnston, 2009; Cai et al., 2015; Curran \& Benton, 2012). In our study, however, repetition of Chinese characters may activate not only the low-level sensory processing, but also the mnemonic processing (Matthews \& Meck, 2016), including phonological and semantic processing. The latter potentially invokes location-independent repetition suppression. As a consequence, the temporal repetition compression remains even when the repeated stimulus changes its location.
The duration compression induced by visual or phonological repetition of Chinese characters replicated and extended earlier research (Birngruber et al., 2015b; Cai et al., 2015; Matthews, 2011, 2015; Schindel et al., 2011). In a recent study by Birngruber et al. (2015b), the subjective duration of repeating strings of consonant was underestimated compared to the novel one in a two-interval paradigm. The effect of repeating consonant strings was similar to the visual repetition. Matthews $(2011,2015)$ found the repetition compression also happened with facial or scenery pictures. But those complex pictures contain multiple visual features and semantic information, which are difficult to be disassociated in their studies. Our study revealed that the repetition of phonological constituent without visual and semantic similarity significantly produced a shorter subjective duration, though the effect was weaker than the pure visual repetition. The results indicate that visual similarity is not a solely determining factor for the repetition compression. Similarly, phonological repetition could activate the "auditory repetition" automatically.

Unlike phonological repetition, semantic similarity failed to affect apparent duration. Although semantic similarity could facilitate explicit word identification (Neely, 1991; Perfetti \& Liu, 2006), the identification task requires a direct semantic processing of the word, which occurs in a late stage compared to visual and phonological information processing (Perfetti \& Liu, 2006). The absence of duration compression for semantic repetition may indicate that the temporal repetition compression is likely modulated at the early stage of processing repetition features in the processing hierarchy.

One proposal for the repetition compression is coding efficiency under the framework of predictive coding (Eagleman \& Pariyadath, 2009; Matthews et al., 2014), which argues repetition suppression depends on the differences between the predicted and the actual signals. The predictive coding framework (Friston, 2005, Rao \& Ballard, 1999) assumes that bottom-up signals are only passed on to the next stage when the information deviates from the top-down predictions. Neural responses are suppressed for the predicted signals and activated for the "surprised" prediction errors. The predictive coding account may offer a unified explanation for the differential repetition effects we found here. In a short sequence, participants tend to keep the same prediction, which plays an important role in the recency effect (Davelaar, Goshen-Gottstein, Ashkenazi, Haarmann, \& Usher, 2005; Glanzer, 1972) and the central tendency effect (Cicchini, Arrighi, Cecchetti, Giusti, \& Burr, 2012; Jazayeri \& Shadlen, 2010; Shi et al., 2013). Similarly, in our duration comparison paradigm, participants likely expect the same stimulus for the second comparison presentation. Prediction error (or "surprise") thus varied across three different repetition conditions: no surprise for the visual repetition, partial surprise (in vision) for the phonological repetition, but both auditory and visual surprise for the semantic repetition. The 
auditory and visual novel information contained in the semantic repetition would be similar to the non-repetition condition. In line with predictive coding, magnitudes of prediction error would qualitatively "predict" differential repetition compression observed in three repetition conditions.

One might argue attentional modulation in a repeated presentation contributes to the duration distortion in the present study. A novel (rare) stimulus often captures attention compared to the repeated one, thus according to the attentiongating theory (Zakay \& Block, 1997) more temporal information is accumulated for the novel one, resulting in a longer perceived duration for the novel than the repeated one (Matthews \& Meck, 2016; Tse et al., 2004). But attentional modulation alone cannot explain our findings of temporal repetition compression. First, earlier studies have shown the repetition may evoke an attentional enhancement of perceptual processing, causing the repeated stimulus appears longer than a novel one (Matthews, 2015; Matthews \& Meck, 2016). Second, shift of spatial attention by the location manipulation (Experiment $1 \mathrm{~b}$ ) deteriorated the discrimination sensitivity, but not the duration compression of repeated stimuli. Thus, attentional modulation, if any, did not play a key role in the temporal repetition compression we observed here.

In conclusion, we found visual- and phonological-repetition, but not semantic repetition, of Chinese characters induced a duration compression, and the repetition compression is location independent. The compression effects are likely due to the repetition suppression in both early sensory processing of visual and phonological (auditory) information. Semantic similarity processing, however, may take place at relative late stage, which did not interact with the timing judgment.

Acknowledgements This work was supported by the Nature Science Foundation of JiangSu Province in China (Grants No. BK20160171) to LJ and German research foundation DFG SH166 3/1 to ZS. The funding institution had no role in the study design, data collection, analysis decision to publish, or preparation of the manuscript.

\section{References}

Allman, M. J., Teki, S., Griffiths, T. D., \& Meck, W. H. (2014). Properties of the internal clock: First- and second-order principles of subjective time. Annual Review of Psychology, 65, 743-771. doi:10.1146/ annurev-psych-010213-115117

Ayhan, I., Bruno, A., Nishida, S., \& Johnston, A. (2009). The spatial tuning of adaptation-based time compression. Journal of Vision, 9(11), 2.1-12. doi:10.1167/9.11.2

Birngruber, T., Schroter, H., \& Ulrich, R. (2015a). Introducing a control condition in the classic oddball paradigm: Oddballs are overestimated in duration not only because of their oddness. Attention, Perception \& Psychophysics, 77(5), 1737-1749. doi:10. 3758/s13414-015-0868-7

Birngruber, T., Schröter, H., \& Ulrich, R. (2015b). The influence of stimulus repetition on duration judgments with simple stimuli. Frontiers in Psychology, 6, 1213. doi:10.3389/fpsyg.2015.01213
Brainard, D. H. (1997). The psychophysics toolbox. Spatial Vision, 10(4), 433-436. doi:10.1163/156856897X00357

Burr, D., Tozzi, A., \& Morrone, M. C. (2007). Neural mechanisms for timing visual events are spatially selective in real-world coordinates. Nature Neuroscience, 10(4), 423-425. doi:10.1038/nn1874

Cai, M., \& Brysbaert, M. (2010). SUBTLEX-CH: Chinese word and character frequencies based on film subtitles. PloS One, 5(6), e10729. doi:10.1371/journal.pone.0010729

Cai, M., Eagleman, D. M., \& Ma, W. (2015). Perceived duration is reduced by repetition but not by high-level expectation. Journal of Vision, 15(13), 19. doi:10.1167/15.13.19

Cicchini, G. M., Arrighi, R., Cecchetti, L., Giusti, M., \& Burr, D. C. (2012). Optimal encoding of interval timing in expert percussionists. Journal of Neuroscience, 32(3), 1056-1060. doi:10.1523/ JNEUROSCI.3411-11.2012

Curran, W., \& Benton, C. P. (2012). The many directions of time. Cognition, 122(2), 252-257. doi:10.1016/j.cognition.2011.10.016

Davelaar, E. J., Goshen-Gottstein, Y., Ashkenazi, A., Haarmann, H. J., \& Usher, M. (2005). The demise of short-term memory revisited: Empirical and computational investigations of recency effects. Psychological Review, 112(1), 3-42. doi:10.1037/0033-295x.112. 1.3

Droit-Volet, S., \& Gil, S. (2009). The time-emotion paradox. Philosophical Transactions of the Royal Society of London. Series B, Biological Sciences, 364(1525), 1943-1953. doi:10.1098/rstb. 2009.0013

Eagleman, D. M., \& Pariyadath, V. (2009). Is subjective duration a signature of coding efficiency? Philosophical Transactions of the Royal Society of London. Series B, Biological Sciences, 364(1525), 1841-1851. doi:10.1098/rstb.2009.0026

Friston, K. (2005). A theory of cortical responses. Philosophical transactions of the Royal Society of London. Series B, Biological Sciences, 360(1456), 815-836. doi:10.1098/rstb.2005.1622

Ganzenmüller, S., Shi, Z., \& Müller, H. J. (2012). Duration reproduction with sensory feedback delay: Differential involvement of perception and action time. Frontiers in Integrative Neuroscience, 6, 95. doi:10. 3389/fnint.2012.00095

Glanzer, M. (1972). Storage mechanisms in recall. In G. H. Bower (Ed.), The psychology of learning and motivation (Vol. 5, pp. 129-193). New York: Academic Press.

Grill-Spector, K., Henson, R., \& Martin, A. (2006). Repetition and the brain: Neural models of stimulus-specific effects. Trends in Cognitive Sciences, 10(1), 14-23. doi:10.1016/j.tics.2005.11.006

Gu, B. M., Jurkowski, A. J., Shi, Z., \& Meck, W. H. (2016). Bayesian optimization of interval timing and biases in temporal memory as a function of temporal context, feedback, and dopamine levels in young, aged and Parkinson's disease patients. Timing \& Time Perception, 4, 315-342. doi:10.1163/22134468-00002072

Henson, R. N. A., \& Rugg, M. D. (2003). Neural response suppression, haemodynamic repetition effects, and behavioural priming. Neuropsychologia, 41(3), 263-270. doi:10.1016/S0028-3932(02) 00159-8

Jazayeri, M., \& Shadlen, M. N. (2010). Temporal context calibrates interval timing. Nature Neuroscience, 13(8), 1020-1026. doi:10.1038/ nn. 2590

Jia, L., Shi, Z., Zang, X., \& Müller, H. J. (2013). Concurrent emotional pictures modulate temporal order judgments of spatially separated audio-tactile stimuli. Brain Research, 1537, 156-163. doi:10.1016/j. brainres.2013.09.008

Jia, L., Shi, Z., \& Feng, W. (2015). Wearing weighted backpack dilates subjective visual duration: the role of functional linkage between weight experience and visual timing. Frontiers in Psychology, 6, 1373. doi:10.3389/fpsyg.2015.01373

Johnston, A., Arnold, D. H., \& Nishida, S. (2006). Spatially localized distortions of event time. Current Biology: $C B, 16(5), 472-479$. doi: 10.1016/j.cub.2006.01.032 
Kim, E., \& McAuley, J. D. (2013). Effects of pitch distance and likelihood on the perceived duration of deviant auditory events. Attention, Perception \& Psychophysics, 75(7), 1547-1558. doi:10.3758/ s13414-013-0490-5

Matthews, W. J. (2011). Stimulus repetition and the perception of time: The effects of prior exposure on temporal discrimination, judgment, and production. PLoS One, 6(5), e19815. doi:10.1371/journal.pone. 0019815

Matthews, W. J. (2015). Time perception: The surprising effects of surprising stimuli. Journal of Experimental Psychology: General, 144(1), 172-197. doi:10.1037/xge0000041

Matthews, W. J., \& Gheorghiu, A. I. (2016). Repetition, expectation, and the perception of time. Current Opinion in Behavioral Sciences, 8 , 110-116. doi:10.1016/j.cobeha.2016.02.019

Matthews, W. J., \& Meck, W. H. (2016). Temporal cognition: Connecting subjective time to perception, attention, and memory. Psychological Bulletin. doi:10.1037/bul0000045

Matthews, W. J., Terhune, D. B., Rijn, H. V., Eagleman, D. M., Sommer, M. A., \& Meck, W. H. (2014). Subjective duration as a signature of coding efficiency: Emerging links among stimulus repetition, predictive coding, and cortical GABA levels. Timing \& Time Perception Reviews, 1(1), 1-11. doi:10.1163/24054496-00101005

Neely, J. H. (1991). Semantic priming effects in visual word recognition: A selective review of current findings and theories. In D. Besner \& G. W. Humphreys (Eds.), Basic processes in reading: Visual word recognition (pp. 264-336). Hillsdale, NJ: Erlbaum.

Pariyadath, V., \& Eagleman, D. (2007). The effect of predictability on subjective duration. PloS One, 2(11), 1-6. doi:10.1371/journal. pone. 0001264

Pariyadath, V., \& Eagleman, D. M. (2008). Brief subjective durations contract with repetition. Journal of Vision, 8(16), 1-6. doi:10. 1167/8.16.11

Pariyadath, V., \& Eagleman, D. M. (2012). Subjective duration distortions mirror neural repetition suppression. PloS One, 7(12), e49362. doi:10.1371/journal.pone.0049362

Perfetti, C. A., \& Liu, Y. (2006). Reading Chinese characters: Orthography, phonology, meaning, and the lexical constituency model. In P. Li, L. H. Tan, E. Bates, \& O. J. L. Tzeng (Eds.), The handbook of east Asian psycholinguistics (Vol. 1. Chinese) (pp. 225236). NY: Cambridge University Press.

Qiao, F., Zheng, L., Li, L., Zhu, L., \& Wang, Q. (2014). Reduced repetition suppression in the occipital visual cortex during repeated negative Chinese personality-trait word processing. Scandinavian Journal of Psychology, 55(6), 533-537. doi:10.1111/sjop.12164

Rao, R. P., \& Ballard, D. H. (1999). Predictive coding in the visual cortex: A functional interpretation of some extra-classical receptive-field effects. Nature Neuroscience, 2(1), 79-87. doi:10.1038/4580
Rose, D., \& Summers, J. (1995). Duration illusions in a train of visual stimuli. Perception, 24(10), 1177-1187. doi:10.1068/p241177

Rouder, J. N., Morey, R. D., Speckman, P. L., \& Province, J. M. (2012). Default Bayes factors for ANOVA designs. Journal of Mathematical Psychology, 56(5), 356-374. doi:10.1016/j.jmp. 2012.08.001

Schacter, D. L., Wig, G. S., \& Stevens, W. D. (2007). Reductions in cortical activity during priming. Current Opinion in Neurobiology, 17(2), 171-176. doi:10.1016/j.conb.2007.02.001

Schindel, R., Rowlands, J., \& Arnold, D. H. (2011). The oddball effect: Perceived duration and predictive coding. Journal of Vision, 11(2), 1-9. doi:10.1167/11.2.17

Sekiguchi, T., Koyama, S., \& Kakigi, R. (2000). The effect of word repetition on evoked magnetic responses in the human brain. Japanese Psychological Research, 42(1), 3-14. doi:10.1111/14685884.00126

Shi, Z., \& Burr, D. (2016). Predictive coding of multisensory timing. Current Opinion in Behavioral Sciences, 8, 200-206. doi:10.1016/ j.cobeha.2016.02.014

Shi, Z., Church, R. M., \& Meck, W. H. (2013). Bayesian optimization of time perception. Trends in Cognitive Sciences, 17(11), 556-564. doi:10.1016/j.tics.2013.09.009

Shi, Z., Ganzenmüller, S., \& Müller, H. J. (2013). Reducing bias in auditory duration reproduction by integrating the reproduced signal. PLoS One, 8(4), e62065. doi:10.1371/journal.pone.0062065

Shi, Z., Jia, L., \& Müller, H. J. (2012). Modulation of tactile duration judgments by emotional pictures. Frontiers in Integrative Neuroscience, 6, 24. doi:10.3389/fnint.2012.00024

Takahiro, S., Sachiko, K., \& Ryusuke, K. (2004). The effect of phonological repetition on cortical magnetic responses evoked by visually presented words. Journal of Cognitive Neuroscience, 16(7), 12501261. doi:10.1162/0898929041920432

Treutwein, B., \& Strasburger, H. (1999). Fitting the psychometric function. Perception \& Psychophysics, 61(1), 87-106. doi:10.3758/ BF03211951

Tse, P. U., Intriligator, J., Rivest, J., \& Cavanagh, P. (2004). Attention and the subjective expansion of time. Perception \& Psychophysics, 66(7), 1171-1189. doi:10.3758/BF03196844

Wheatley, T., Weisberg, J., Beauchamp, M., \& Martin, A. (2005). Automatic priming of semantically related words reduces activity in the fusiform gyrus. Journal of Cognitive Neuroscience, 17(12), 1871-1885. doi:10.1162/089892905775008689

Xuan, B., Zhang, D., He, S., \& Chen, X. (2007). Larger stimuli are judged to last longer. Journal of Vision, 7(10), 1-5. doi:10.1167/7.10.2

Zakay, D., \& Block, R. A. (1997). Temporal cognition. Current Directions in Psychological Science, 6(1), 12-16. doi:10.1111/ 1467-8721.ep11512604 